International Mathematical Forum, Vol. 9, 2014, no. 11, 515 - 522

HIKARI Ltd, www.m-hikari.com

http://dx.doi.org/10.12988/imf.2014.414

\title{
Surjective Maps Preserving Local Spectral Radius
}

\author{
Mustapha Ech-Cherif El Kettani \\ Department of Mathematics \\ Faculty of Sciences DharMahraz Fes \\ B.P. 1796 Atlas Fes, Morocco \\ Hassane Benbouziane \\ Department of Mathematics \\ Faculty of Sciences DharMahraz Fes \\ B.P. 1796 Atlas Fes, Morocco
}

Copyright (c) 2014 Mustapha Ech-Cherif El Kettani and Hassane Benbouziane. This is an open access article distributed under the Creative Commons Attribution License, which permits unrestricted use, distribution, and reproduction in any medium, provided the original work is properly cited.

\begin{abstract}
Let $\mathcal{B}(X)$ be the algebra of all bounded linear operators on a complex Banach space $X$. Let $x_{0}$ is a nonzero fixed vector in $X$. We give the concrete form of every surjective map $\phi$ from $\mathcal{B}(X)$ into its self, such that the local spectral radius of $\phi(T) \phi(S)+\phi(R)$ at $x_{0}$ equals the local spectral radius of $T S+R$ at $x_{0}$. We do not assume $\phi$ to be linear, or even additive.
\end{abstract}

Mathematics Subject Classification: Primary 47A11; Secondary 47A10, 47B48

Keywords: local spectral radius, local spectral, preserver problems

\section{Introduction}

Throughout this note, $X$ and $Y$ will denote a infinite-dimensional complex Banach spaces, and $\mathcal{B}(X)$ the algebra of all linear bounded operators on $X$ and its unit will be denoted by $I$. The duality between the Banach space $X$ 
and its dual, $X^{*}$, will be denoted by $\langle.,$.$\rangle . For x \in X$ and $f \in X^{*}$, we denote as usual by $x \otimes f$ the rank one operator on $X$ given by $z \mapsto\langle z, f\rangle x$. We say that a map $\phi: \mathcal{B}(X) \rightarrow \mathcal{B}(X)$ is unital if $\phi(I)=I$. The local resolvent of an operator $T \in \mathcal{B}(X)$ at some point $x \in X$, denoted by $\rho_{T}(x)$, is the union of all open subsets $U \subseteq \mathbb{C}$ for which there exists an analytic function $f: U \rightarrow X$ such that $(T-\lambda) f(\lambda)=x$ for all $\lambda \in U$.

The local spectrum of $T$ at $x$ is defined as the complement in $\mathbb{C}$ of the local resolvent $\rho_{T}(x)$, i.e $\sigma_{T}(x)=\mathscr{C} \backslash \rho_{T}(x)$. Let us observe that $\sigma_{T}(x)$ is a (possibly empty) closed subset of $\sigma(T)$, the usual spectrum of $T \in \mathcal{B}(X)$. The local spectral radius of $\mathrm{T}$ at $\mathrm{x}$ is defined by

$$
r_{T}(x)=\limsup _{n \rightarrow \infty}\left\|T^{n} x\right\|^{\frac{1}{n}}
$$

An operator $T \in \mathcal{B}(X)$ is said to have the single-valued extension property at $\lambda_{0} \in \mathbb{C}$, abbreviated T has the SVEP at $\lambda_{0}$, if for every neighbourhood $U$ of $\lambda_{0}$ the only analytic function $f: U \rightarrow X$ which satisfies the equation

$$
(\lambda-T) f(\lambda)=0 \quad(\lambda \in U)
$$

is the constant function $f \equiv 0$. The operator $T$ is said to have the SVEP if $T$ has the SVEP at every $\lambda \in \mathbb{C}$.

Evidently, T has SVEP at every $\lambda \in \mathbb{C} \backslash \operatorname{int}\left(\sigma_{p}(T)\right)$, where $\operatorname{int}\left(\sigma_{p}(T)\right)$ denotes the interior of the point spectrum of $T$. In particular, if $\sigma_{p}(T)$ has empty interior, then $T$ has SVEP. For example, every finite rank operator has SVEP.

In the finite dimensional case, we denote by $\mathcal{M}_{n}$ the algebra of all complex $n \times n$ matrices. Every $T$ belonging to $\mathcal{M}_{n}$ has the single-valued extension property. We can see, for example, the books $[1,15]$ for further information on the local spectral theory.

The problem of characterizing linear or additive maps on operator algebras preserving certain properties, subsets or relations has attracted the attention of many authors in the last decades (see for instance $[2,4,5,9,10,13]$ ).

In [5], Bourhim and Ransford studied additive maps on $\mathcal{B}(X)$ preserving the local spectrum at each point $x \in X$. They showed that the only additive $\operatorname{map} \phi: \mathcal{B}(X) \rightarrow \mathcal{B}(X)$ for which

$$
\sigma_{T}(x)=\sigma_{\phi(T)}(x) \quad(x \in X, T \in \mathcal{B}(X))
$$

is the identity on $\mathcal{B}(X)$.

J. Bračič and V. Müller in [7] characterized the surjective linear map $\phi$ : $\mathcal{B}(X) \rightarrow \mathcal{B}(X)$ satisfies $r_{T}(x)=r_{\phi(T)}(x)$ for all $T \in \mathcal{B}(X)$ and $x$ is a nonzero vector fixed in $X$. Different versions of this result in the case when $X$ is finitedimensional were considered in [4]. In the infinite-dimensional case, the linear, 
surjective and continuous maps preserving different local spectral properties were characterized in [2].

in recent years a great activity has occurred in the question of relaxing the assumption of linearity or additivity (see, for example, $[3,6,8,11,12]$ ).

In [3], Bourhim and Mashreghi described surjective maps on $\mathcal{B}(X)$ which preserve the local spectral radius distance zero at points of $X$. They showed, in particular, that if $\phi$ is a surjective map on $\mathcal{B}(X)$ such that for every $x \in X$

$$
r_{T-S}(x)=0 \text { if and only if } r_{\phi(T)-\phi(S)}(x)=0,(T, S \in \mathcal{B}(X)) \text {, }
$$

then there exists a nonzero scalar $c$ and an operator $A \in \mathcal{B}(X)$ such that $\phi(T)=c T+A$ for all $T \in \mathcal{B}(X)$.

While, Different versions of this result in the case where $X$ is finite-dimensional and $x$ is a nonzero fixed vector were considered in [6] and [11].

C. Costara in [11], showed the following result holds true without the linearity or additivity of the map.

Theorem 1.1 ([11, Theorem 1.2]) Let $x_{0}$ be a non-zero vector in $\mathbb{C}^{n}$ and let $\phi: \mathcal{M}_{n} \rightarrow \mathcal{M}_{n}$ be a surjective map with $\phi(0)=0$ such that

$$
r_{T+S}\left(x_{0}\right)=r_{\phi(T)+\phi(S)}\left(x_{0}\right) \quad\left(T, S \in \mathcal{M}_{n}\right)
$$

then there exists a unimodular $\alpha \in \mathbb{C}$ and an invertible matrix $A \in \mathcal{M}_{n}$ such that either $A x_{0}=x_{0}$ and

$$
\phi(T)=\alpha A T A^{-1}, \text { for }, \text { all },\left(T \in \mathcal{M}_{n}\right)
$$

or $A \overline{x_{0}}=x_{0}$ and

$$
\phi(T)=\alpha A \bar{T} A^{-1}, \text { for }, \text { all },\left(T \in \mathcal{M}_{n}\right) .
$$

Where the matrix $\bar{T}$ is obtained from $T$ by entrywise complex conjugation.

The purpose of this note is to study the same problem as in [3] but at nonzero vector fixed $x_{0}$ in $X$, with respect to the ring structure of the algebra. More precisely, we will show that if a surjective maps (not necessarily additive), $\phi: \mathcal{B}(X) \rightarrow \mathcal{B}(X)$ satisfies

$$
r_{T S+R}\left(x_{0}\right)=r_{\phi(T) \phi(S)+\phi(R)}\left(x_{0}\right) \quad T, S, R \in \mathcal{B}(X)
$$

if and only if there exists an invertible bounded linear or conjugate linear operator $A: X \longrightarrow X$ such that $\phi(T)=A T A^{-1}$ for all $T \in \mathcal{B}(X)$. We also give the version result in the finite dimensional case. 


\section{Preliminaries and Notations}

In this section, we collect some lemmas that are needed for the proof of our main results. The first one plays a key role in the proof of Theorem 3.1. It is inspired [3, Theorem 3.1] in which the authors have obtained a local version of Zemánek's spectral characterization of the radical.

Lemma 2.1 Let $x_{0}$ be a nonzero vector in $X$. Let $T, S \in \mathcal{B}(X)$ such that $r_{T+R}\left(x_{0}\right)=r_{S+R}\left(x_{0}\right)$ for every $R \in \mathcal{B}(X)$ then $T=S$.

Proof. Let $T, S \in \mathcal{B}(X)$ such that $r_{T+R}\left(x_{0}\right)=r_{S+R}\left(x_{0}\right)$ for every $R \in$ $\mathcal{B}(X)$. If we denote $A=T-S$ then $r_{A+R}\left(x_{0}\right)=r_{T-S+R}\left(x_{0}\right)=r_{R}\left(x_{0}\right)$ for all $R \in \mathcal{B}(X)$. By [3, Theorem 3.1], it follows that $A=0$, as desired.

We shall make extensive use of the following result.

Lemma 2.2 ([1, Theorem 2.22])

Let $T \in \mathcal{B}(X)$, let $\lambda \in \mathbb{C}^{\prime}$ and let $x \in \operatorname{ker}(T-\lambda)$. Then $\sigma_{T}(x) \subset\{\lambda\}$. If, further, $x \neq 0$ and $T$ has SVEP at $\lambda$, then $\sigma_{T}(x)=\{\lambda\}$.

An operator $P \in \mathcal{B}(X)$ is called an idempotent operator if $P^{2}=P$. We denote by $\mathcal{P}(X)=\left\{P \in \mathcal{B}(X): P^{2}=P\right\}$ the set of all idempotent operators. The following lemma was obtained in [12], Theorem 1.1, in which the authors have given the form of every unital surjective map $\phi$ on $\mathcal{B}(X)$ such that $T S$ is a nonzero idempotent if and only if $\phi(T) \phi(S)$ is for all $T, S \in \mathcal{B}(X)$ when the dimension of $X$ is at least 3 .

Lemma 2.3 ([12, Theorem 1.1])

Let $X$ be an infinite dimensional Banach space. Then a unital surjective map $\phi: \mathcal{B}(X) \rightarrow \mathcal{B}(X)$ satisfies that

$$
T S \in \mathcal{P}(X) \backslash\{0\} \Leftrightarrow \phi(T) \phi(S) \in \mathcal{P}(X) \backslash\{0\} \quad \text { for all } T, S \in \mathcal{B}(X)
$$

if and only if there exists an invertible bounded linear or conjugate-linear operator $A: X \longrightarrow X$ such that $\phi(T)=A T A^{-1}$ for all $T \in \mathcal{B}(X)$.

\section{Main Results}

We begin this section with the following result which is the main theorem. Our arguments are influenced by the ones given in [14].

Theorem 3.1 Let $x_{0}$ be a nonzero vector of $X$. A surjective map $\phi$ : $\mathcal{B}(X) \rightarrow \mathcal{B}(X)$ satisfies

$$
r_{T S+R}\left(x_{0}\right)=r_{\phi(T) \phi(S)+\phi(R)}\left(x_{0}\right) \quad T, S, R \in \mathcal{B}(X),
$$

if and only if there exists an invertible bounded linear or conjugate-linear operator $A: X \longrightarrow X$ such that $A x_{0}=x_{0}$ and $\phi(T)=A T A^{-1}$ for all $T \in \mathcal{B}(X)$. 
Proof. Let $A: X \longrightarrow X$ be an invertible bounded linear or conjugate-linear operator such that $A x_{0}=x_{0}$. By [2, Lemma 2.3], we have

$$
r_{T S+R}\left(x_{0}\right)=r_{A(T S+R) A^{-1}}\left(A x_{0}\right)=r_{A(T S+R) A^{-1}}\left(x_{0}\right),
$$

for every $T, S, R \in \mathcal{B}(X)$. This proves the "if" part.

To prove the "only if" part, assume that $\phi$ is a surjective map from $\mathcal{B}(X)$ into $\mathcal{B}(X)$ satisfying $(4)$, and let us first prove that $\phi(0)=0$ and $r_{\phi(T)}\left(x_{0}\right)=$ $r_{T}\left(x_{0}\right)$ for every $T \in \mathcal{B}(X)$. Let $T, R \in \mathcal{B}(X)$, we have

$$
r_{\phi(T) \phi(0)+\phi(R)}\left(x_{0}\right)=r_{R}\left(x_{0}\right)=r_{\phi(0) \phi(T)+\phi(R)}\left(x_{0}\right) .
$$

Since $\phi$ is surjective, then by Lemma $2.1 \phi(0) \phi(T)=\phi(T) \phi(0)$. Therefore $\phi(0)$ commutes with every operator in $\mathcal{B}(X)$. Hence there exist $\lambda \in \mathbb{C}$ such that $\phi(0)=\lambda I$. Indeed, let $R=\phi(0)$. It is sufficient to prove that given any $x \in X$ we have that $x$ and $R x$ are linearly dependent. If this were not true, $R x \neq 0$ and we could find a linear functional $f \in X^{*}$ such that $f(x)=1$ and $f(R x)=0$. Then for $T:=x \otimes f$ we have $R T x=R x \neq 0$ but $T R x=0$. This contradiction shows that $\phi(0)=\lambda I$ for some scalar $\lambda \in \mathscr{C}$. Moreover,

$$
r_{\lambda \phi(T)+\phi(R)}\left(x_{0}\right)=r_{R}\left(x_{0}\right)=r_{\lambda \phi(S)+\phi(R)}\left(x_{0}\right) \text { for every } R \in \mathcal{B}(X) .
$$

So, by Lemma $2.1, \lambda \phi(T)=\lambda \phi(S)$ for every $T, S \in \mathcal{B}(X)$, from which it follows that $\lambda=0$ and $\phi(0)=0$.

And hence we infer that

$$
r_{\phi(T)}\left(x_{0}\right)=r_{T}\left(x_{0}\right),(T \in \mathcal{B}(X)) .
$$

Next, we show that $\phi(I)=I$. Similar to the previous, for $T, R \in \mathcal{B}(X)$, we have

$$
r_{\phi(T) \phi(I)+\phi(R)}\left(x_{0}\right)=r_{T+R}\left(x_{0}\right)=r_{\phi(I) \phi(T)+\phi(R)}\left(x_{0}\right)
$$

then by Lemma $2.1 \phi(I) \phi(T)=\phi(T) \phi(I)$. Thus $\phi(I)$ commutes with every operator in $\mathcal{B}(X)$. This show that $\phi(I)=\alpha I$ for some scalar $\alpha$.

By $(5), r_{\phi(T)}\left(x_{0}\right)=r_{I T}\left(x_{0}\right)=r_{\alpha \phi(T)}\left(x_{0}\right)$ for every $T \in \mathcal{B}(X)$. Then $r_{\phi(T)}\left(x_{0}\right)=|\alpha| r_{\phi(T)}\left(x_{0}\right)$. Since $\phi$ is surjective we can find an operator $T \in$ $\mathcal{B}(X)$ that satisfies $r_{\phi(T)}\left(x_{0}\right)=1$ (take for instance $\phi(T)=x_{0} \otimes f$ such that $\left.f\left(x_{0}\right)=1\right)$, then $|\alpha|=1$.

On the other hand, $r_{(\alpha+1) \phi(T)}\left(x_{0}\right)=r_{\phi(I) \phi(T)+\phi(T)}\left(x_{0}\right)=r_{2 T}\left(x_{0}\right)$. Hence $|\alpha+1| r_{T}\left(x_{0}\right)=2 r_{T}\left(x_{0}\right)$. And for the same reason $|\alpha+1|=2$. Since $|\alpha|=1$ and $|\alpha+1|$ is the distance between $\alpha$ and -1 , it follows that $\alpha=1$. This shows that $\phi(I)=I$. And hence we infer that

$$
\left.r_{T+S}\left(x_{0}\right)=r_{\phi(T)+\phi(S)}\left(x_{0}\right) \text {, for every } T, S \in \mathcal{B}(X)\right) \text {. }
$$


Now, we show that $\phi$ is injective. To do that, let $T, S \in \mathcal{B}(X)$ such that $\phi(T)=\phi(S)$. For every $R \in \mathcal{B}(X)$ we have, by (6),

$$
r_{T+R}\left(x_{0}\right)=r_{\phi(T)+\phi(R)}\left(x_{0}\right)=r_{\phi(S)+\phi(R)}\left(x_{0}\right)=r_{S+R}\left(x_{0}\right)
$$

then, by Lemma $2.1 T=S$, as desired.

It remains to show that $\phi$ takes the desired form. First let $T, S \in \mathcal{B}(X)$, combining (4) and (6), we get that

$$
r_{\phi(T S)+\phi(R)}\left(x_{0}\right)=r_{T S+R}\left(x_{0}\right)=r_{\phi(T) \phi(S)+\phi(R)}\left(x_{0}\right)
$$

for every $R \in \mathcal{B}(X)$. Hence, by Lemma 2.1

$$
\phi(T S)=\phi(T) \phi(S)
$$

For $P \in \mathcal{B}(X)$ we can affirm that $P \in \mathcal{P}(X)$ if and only if $\phi(P) \in \mathcal{P}(X)$.

Indeed, let $P \in \mathcal{P}(X)$ i.e $P^{2}=P$ then, by $(7), \phi(P)^{2}=\phi\left(P^{2}\right)=\phi(P)$ hence $P \in \mathcal{P}(X)$. Since $\phi$ is bijective, the reverse implication is the same way.

Since $\phi$ is unital bijective multiplicative map, we can show without any effort that $T S \in \mathcal{P}(X) \backslash\{0\} \Leftrightarrow \phi(T) \phi(S) \in \mathcal{P}(X) \backslash\{0\}$ for every $T, S \in \mathcal{B}(X)$. Lemma 2.3 implies that there exists an invertible bounded linear or conjugatelinear operator $A: X \longrightarrow X$ such that $\phi(T)=A T A^{-1}$ for all $T \in \mathcal{B}(X)$.

Finally let us prove that $A x_{0}=x_{0}$. Assume on the contrary that $A^{-1} x_{0}$ and $x_{0}$ are linearly independent. We could find $f \in X^{*}$ such that $f\left(A^{-1} x_{0}\right)=0$ and $f\left(x_{0}\right)=1$. Then for $T:=x_{0} \otimes f$ we have $T x_{0}=x_{0}$. Thus, $r_{T}\left(x_{0}\right)=1$. On the other hand $A T A^{-1} x_{0}=0$ then $r_{\phi(T)}\left(x_{0}\right)=0$, arriving to a contradiction. We conclude that $A x_{0}=\mu x_{0}$ for some nonzero scalar $\mu$.

Dividing $A$ by $\mu$ if necessary, we may assume that $A x_{0}=x_{0}$. This finishes the proof.

Next we give the version of Theorem 3.1 in the case of two different Banach spaces.

Corollary 3.2 Let $x_{0}$ be a nonzero vector of $X$ and $y_{0}$ in $Y$. If $\phi: \mathcal{B}(X) \rightarrow$ $\mathcal{B}(Y)$ is a surjective map satisfying

$$
r_{T S+R}\left(x_{0}\right)=r_{\phi(T) \phi(S)+\phi(R)}\left(y_{0}\right) \quad T, S, R \in \mathcal{B}(X)
$$

then there exists an invertible bounded linear or conjugate-linear operator $A$ : $X \longrightarrow Y$ such that $A x_{0}=y_{0}$ and $\phi(T)=A T A^{-1}$ for all $T \in \mathcal{B}(X)$.

Proof. We can find a bijective map $R_{0} \in \mathcal{B}(X, Y)$ such that $R_{0}\left(x_{0}\right)=y_{0}$. Consider $\psi: \mathcal{B}(X) \rightarrow \mathcal{B}(X)$ defined by $\psi: T \mapsto R_{0}^{-1} \phi(T) R_{0}$ for all $T \in \mathcal{B}(X)$.

By $\left[2\right.$, Lemma 2.3] we have $r_{\psi(T)}\left(x_{0}\right)=r_{R_{0}^{-1}(\phi(T)) R_{0}}\left(x_{0}\right)=r_{\phi(T)}\left(R_{0} x_{0}\right)$, for every $T \in \mathcal{B}(X)$. And therefore, by (8),

$$
r_{\psi(T) \psi(S)+\psi(R)}\left(x_{0}\right)=r_{\phi(T) \phi(S)+\phi(R)}\left(R_{0}\left(x_{0}\right)\right)=r_{T S+R}\left(x_{0}\right)
$$


for every $T, S, R \in \mathcal{B}(X)$. So $\psi$ is a surjective map satisfying (4), hence by Theorem 3.1, there exists an invertible bounded linear or conjugate-linear operator $A_{0}: X \longrightarrow X$ such that $A_{0}\left(x_{0}\right)=x_{0}$ and $\psi(T)=A_{0} T A_{0}^{-1}$ for all $T \in \mathcal{B}(X)$. The result follows by taking $A=R_{0} A_{0}$.

Next, we state our main result for the finite dimensional case.

Theorem 3.3 Let $x_{0}$ be a nonzero vector in $\mathbb{C}^{n}$. If $\phi: \mathcal{M}_{n} \rightarrow \mathcal{M}_{n}$ is a surjective map satisfying

$$
r_{T S+R}\left(x_{0}\right)=r_{\phi(T) \phi(S)+\phi(R)}\left(x_{0}\right) \quad\left(T, S, R \in \mathcal{M}_{n}\right),
$$

then there exists an invertible matrix $A \in M_{n}$ such that either $A x_{0}=x_{0}$ and

$$
\phi(T)=A T A^{-1}, \text { for }, \text { all },\left(T \in \mathcal{M}_{n}\right)
$$

or $A \overline{x_{0}}=x_{0}$ and

$$
\phi(T)=A \bar{T} A^{-1}, \text { for, all, }\left(T \in \mathcal{M}_{n}\right) .
$$

Where the matrix $\bar{T}$ is obtained from $T$ by entrywise complex conjugation.

Proof. we will argue as in the proof of Theorem 3.1, So we get $\phi$ is a surjective map such that $\phi(0)=0$ satisfying the assumptions of Theorem 1.1, hence $\phi$ is either of the form (2), or of the form (3). Since $\phi(I)=I$ then $\alpha=1$ and consequently we get the desired result.

\section{References}

[1] P. Aiena, Fredholm and local spectral theory, with applications to multipliers, Kluwer, Dordrecht (2004)

[2] A. Bourhim, Surjective linear maps preserving local spectra, Linear Algebra Appl, 432 (2010), 383-393.

[3] A. Bourhim and J. Mashreghi, Local Spectral Radius Preservers, Integr.Equ. Oper. Theory, 76 (2013), 95-104.

[4] A. Bourhim and V.G Miller, Linear maps on Mn preserving the local spectral radius, Studia Math, 188(1) (2008), 67-75.

[5] A. Bourhim and T.J. Ransford, Additive maps preserving local spectrum, Integr. Equ. Oper. Theory, 55 (2006), 377-385. 
[6] M. Bendaoud, M. Douimi and M. Sarih, Maps on Matrices preserving local spectra, Linear Multilinear Algebra, (2012).http://dx. DOI:10.1080/03081087.2012.716429.

[7] J. Bračič and V. Müller, Local spectrum and local spectral radius of an operator at a fixed vector, Studia Math, 194 (2009), 155-162.

[8] R. Bhatia, P. Šemrl and A.R. Sourour, Maps on matrices that preserve the spectral radius distance, Studia Math, 134 (1999), 99110.

[9] C. Costara, Automatic continuity for linear surjective mappings decreasing the local spectral radius at some fixed vector, Arch. Math, 95(6) (2010), 567-573.

[10] C. Costara, Linear maps preserving operators of local spectral radius zero, Integr. Equ. Oper. Theory, 71(1) (2012), 7-16.

[11] C. Costara, Surjective maps on matrices preserving the local spectral radius distance, Integr. Linear and Multilinear Algebra, (2013), http://dx.DOI:10.1080/03081087.2013.801967.

[12] L. Fang, G. Ji, Y. Pang, Maps preserving the idempotency of products of operators, Linear Algebra Appl. 426 (2007). 40-52.

[13] M. González and M. Mbekhta, Linear maps on Mn preserving the local spectrum, Linear Algebra Appl, 426 (2007), 176-182.

[14] S. O. Kim, Determinant And Spectrum Preserving Maps On Mn, Korean J. Math, 20 (2012), No. 3, pp. 285-291.

[15] K.B. Laursen and M. M. Neumann, An introduction to local spectral theory, Oxford University Press, New York, 2000.

\section{Received: January 5, 2014}

